\title{
Uso de anclajes FRP en vigas de concreto armado reforzados externamente con bandas CFRP sometidas a cargas cíclicas
}

\section{Use of FRP Anchorages in Reinforced Concrete Beams Externally Strengthened with CFRP Bands Subjected to Cyclic Loads}

\author{
Jorge L. Bazán \\ Ingeniero Investigador. \\ Universidad Nacional de Ingeniería, Lima, Perú. \\ jlbazans@uni.pe \\ ORCID: https://orcid.org/0000-0002-7451-0359 \\ Juan D. Samaniego \\ Alumno de posgrado. \\ Pontificia Universidad Católica del Perú, Lima, Perú. \\ diego.samaniego@pucp.edu.pe \\ ORCID: https://orcid.org/0000-0003-3357-0790 \\ Víctor I. Fernández-Dávila \\ Profesor Asociado. \\ Pontificia Universidad Católica del Perú, Lima, Perú. \\ vfernandezdavila@pucp.edu.pe \\ ORCID: https://orcid.org/0000-0002-1333-8989
}

Recibido: 28 de noviembre 2019

Aceptado: 27 de abril 2020

\section{Resumen}

En Perú existe una demanda creciente de mejorar, ampliar o remodelar la infraestructura existente que generalmente ha sido diseñada con estándares estructurales menos exigentes que los actuales. Es por esta razón que se utilizan diversas técnicas entre las que se destaca el reforzamiento mediante sistemas FRP (Fiber Reinforced Polymer). Entre las aplicaciones predominantes de este sistema, se tiene el refuerzo a flexión de elementos de concreto armado tales como muros, losas y, en especial, vigas. Durante la aplicación del FRP, se pueden presentar diversas interferencias o limitaciones constructivas, lo que ha dado lugar al planteamiento de soluciones prácticas y modernas como el uso de sistemas de bandas, platinas y barras de FRP, sistemas del tipo NSM y el uso de anclajes de FRP. Estos procedimientos están contemplados en la guía ACI 440.2R, utilizada para la construcción y el diseño de refuerzo externo con FRP. Este artículo tiene por objetivo mostrar los resultados sobre la experiencia en laboratorio de cuatro vigas de concreto armado, considerando que solamente tres fueron externamente reforzadas a flexión e incluyendo el uso especial de anclajes de FRP con la finalidad de desarrollar el comportamiento a tracción del FRP como refuerzo externo. Los especímenes se sometieron a cargas cíclicas mediante un ensayo cuasi-estático. Los resultados indican que estos elementos, reforzados con bandas y anclajes FRP, poseen una capacidad de resistencia superior a la capacidad nominal a flexión. Además, el comportamiento exhibido durante el ensayo de cada espécimen estuvo bajo control y dentro de los parámetros de seguridad habituales. 


\title{
Palabras clave:
}

Refuerzo FRP, anclaje FRP, comportamiento experimental, vigas de concreto armado, carga cíclica, comportamiento flexural

\begin{abstract}
In Peru, there is a growing demand to improve, expand, or remodel existing infrastructure, which has generally been designed with structural standards less demanding than the current ones. It is for this reason that various techniques are used such as reinforcement using FRP (fiber reinforced polymer) systems. Among the predominant applications of this system is the reinforcement of bending of reinforced concrete elements such as walls, slabs, and especially the beams. During the application of the FRP, various interferences or constructive limitations could occur, which has led to the approach of practical and modern solutions such as the use of FRP band systems, FRP plates and bars, NSM systems, and the use of anchors made of FRP. These procedures are contemplated in the ACI's guide 440.2R (ACI, 2017), used for the construction and design of external reinforcement with FRP on reinforced concrete elements. The objective of this article is to show the results of the laboratory experience from four reinforced concrete beams, considering that only three of them were externally strengthened to bending, and they also included details with anchors made of FRP in order to allow the tensile behavior of the external reinforcement FRP bands. The tested samples were subjected to cyclic loads by a quasi-static test, and the results indicate that these elements strengthened with FRP bands and anchors have a greater resistance capacity than the nominal flexural capacity. Furthermore, the behavior exhibited during the test of each specimen was under control and within the usual safety parameters.
\end{abstract}

Keywords:

FRP reinforcement, FRP anchorage, experimental behavior, reinforced concrete beams, cyclic loads, flexural behavior 


\section{INTRODUCCIÓN}

Las bandas de FRP están conformadas por filamentos de polímero tales como carbono (CFRP), vidrio (GFRP) u otro material sintético. Posteriormente son apiladas (generalmente en una dirección), en forma de banda (tela), platina o barra de sección rectangular o circular y luego son laminadas mediante una resina para estabilizar y constituir este material compuesto. De igual manera, los anclajes FRP son materiales compuestos que están conformados por filamentos sintéticos que se disponen longitudinalmente formando una barra cilíndrica. Esta es curada con resina, ya sea previamente a la instalación al interior de la perforación respectiva o una vez instalada al interior de la perforación. El comportamiento de este sistema depende principalmente de muchas variables, por ejemplo: el diámetro del anclaje, profundidad empotrada, la calidad del concreto, entre otras (Kim \& Smith, 2009).

Estos materiales tienen una variedad de ventajas constructivas en comparación a la tradicional barra de acero de refuerzo. Entre las ventajas más significativas se cuenta con su elevada resistencia química, en especial al ion cloruro (Landesmann, Seruti \& Batista, 2015; Noorunnisa et al., 2010); adicional a ello, el FRP posee baja densidad y una alta resistencia a la tracción (Breña et al., 2001), demostrando su factibilidad y eficiencia en elevar las resistencias finales a flexión y a corte al reforzar vigas de concreto armado (Tan \& Mathilovi, 1998; Bazan \& Fernandez, 2019; Mostofinejad \& Khozaei, 2015). Los resultados de 23 ensayos de flexión en cuatro puntos en vigas de concreto armado (CA) con refuerzo externo CFRP, sometidas a cargas monotónicas hasta la falla, indican que un $70 \%$ de las muestras exhiben una resistencia superior al valor nominal predicho por la guía ACI 440.2R (Bazan \& Fernandez, 2019). Sin embargo, las vigas externamente reforzadas con FRP exhiben un comportamiento más frágil y de considerable menor ductilidad en comparación con aquellas vigas tradicionales de CA sin reforzar externamente (White, Soudki \& Erki, 1999; Mostofinejad y Khozaei, 2015).

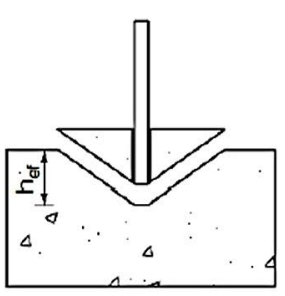

(a) Concrete cone failure

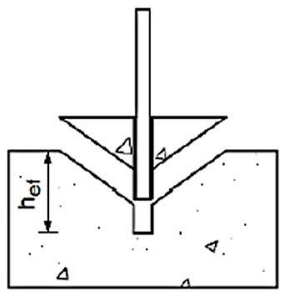

(b) Adhesive-to-concrete interface failure

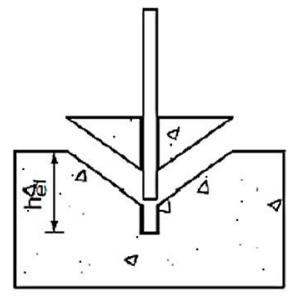

(c) Anchor-to-adhesive interface failure (Combined failure)

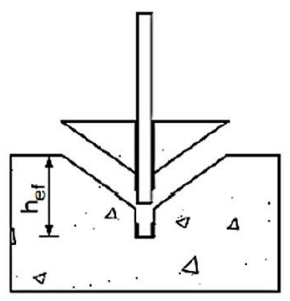

(d) Mixed interface failure (Combined failure)

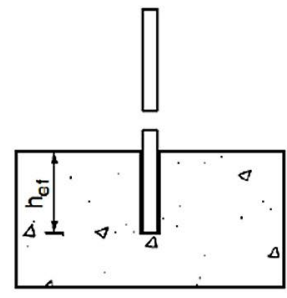

(e) Anchor failure

Figura 1. Modos de falla típicos para anclajes de FRP

Fuente: Kim y Smith (2009)

Actualmente, los anclajes de FRP son empleados como solución constructiva en diferentes elementos estructurales tales como vigas de CA (Orton, Jirsa \& Bayrak, 2008), losas de CA y muros de albañilería (Binici, Ozcebe \& Ozcelik, 2007). Kim y Smith (2010) identificaron experimentalmente 
los siguientes modos de falla de los anclajes FRP: falla de cono de concreto, falla por adherencia de la resina, falla combinada por cono y adherencia, y falla por ruptura del anclaje FRP (Figura 1). En este artículo se presentan los principales resultados obtenidos sobre la aplicación de anclajes para empotrar las bandas de CFRP como refuerzo externo de una viga de CA. Las resistencias de los anclajes utilizados en este trabajo se basan en los valores recomendados en el estudio estadístico realizado por Kim y Smith (2010).

\section{PROGRAMA EXPERIMENTAL}

A continuación, se describen los aspectos generales realizados para la ejecución del programa experimental (Samaniego, 2020).

\subsection{Especímenes}

Se fabricaron cuatro especímenes de vigas de CA con su respectivo bloque de anclaje, las cuales fueron posteriormente reforzadas con bandas y anclajes de FRP de fibra de carbono (CFRP). Todas las barras de acero de refuerzo tienen calidad grado 60 (ASTM A615) con $f_{y}=420 \mathrm{MPa}$, el concreto tiene una calidad $f^{\prime}{ }_{c}=21 \mathrm{MPa}$ y el FRP corresponde a fibra de carbono de la patente Tyfo SCH41. Las propiedades de los materiales suministradas por los fabricantes se muestran en la Tabla 1. La geometría de los especímenes y el refuerzo colocado se muestran en la Figura 2. Del mismo modo, las características del acero de refuerzo, del refuerzo externo longitudinal (LR), transversal (TR) y sus anclajes se muestran en la Tabla 2. Nótese que las vigas V-Control y VE-01 tienen la misma cuantía de acero de refuerzo y difieren únicamente en el refuerzo externo con banda FRP, del mismo modo para los especímenes VE-02 y VE-03.

\subsection{Instrumentación y ensayo de laboratorio}

Los especímenes de CA fueron sometidos a un ensayo cíclico cuasi-estático (carga, descarga y carga en reversa) controlado por desplazamiento lateral según el protocolo I de ensayo del FEMA 461 (2007). El ensayo cíclico cuasi-estático se realizó en el Laboratorio de Estructuras Antisísmicas de la Pontificia Universidad Católica del Perú. Este ensayo consistió en la aplicación de desplazamiento lateral incremental en veinte (20) ciclos, distribuidos a través de diez (10) fases de amplitud. En cada fase de amplitud, se repitieron dos ciclos de carga (Figura 3) y se realizó en forma incremental hasta llegar a la amplitud objetivo $\left(\Delta_{\text {máx }}\right)$.

En este trabajo se utilizaron los desplazamientos laterales $(\Delta)$ y las cargas laterales $(P)$ que fueron medidos por los sensores ubicados en el LDVT y la celda de carga, ubicados a una distancia $H$ de la base (Figura 4). Conforme a lo dispuesto, la viga ensayada se comportó a flexión de manera semejante a una viga en voladizo empotrada en su base y cuyo momento puede calcular como el producto de la carga en el extremo libre y su altura $(M=P \cdot H)$. Notar que el valor de $\mathrm{P}$ puede tomar valores positivos y negativos. Las Figuras 5 y 6 presentan, en una vista general del ensayo, la disposición de los especímenes y los instrumentos como la celda de carga, los LDVTs, la computadora y el Datalogger para adquisición de datos. 
Tabla 1. Propiedades de los materiales empleados

\begin{tabular}{lcccccc}
\hline \multicolumn{1}{c}{ ID } & $\begin{array}{c}f^{\prime}{ }^{c} \\
(\mathrm{MPa})\end{array}$ & $\begin{array}{c}f_{y} \\
(\mathrm{MPa})\end{array}$ & FRP & $\begin{array}{c}E_{f} \\
(\mathrm{GPa})\end{array}$ & $\begin{array}{c}\varepsilon_{f u} \\
(\%)\end{array}$ & $\begin{array}{c}t_{f} \\
(\mathrm{~mm})\end{array}$ \\
\hline V-Control & 21 & 420 & - & - & - & - \\
VE-0X & 21 & 420 & Tyfo SCH-41 & 95.8 & 1.00 & 1.0 \\
\hline$\{-\}$ & $\begin{array}{l}\text { No hay valor } \\
t_{f}\end{array}$ & $:$ Espesor de la banda de CFRP \\
$E_{f}$ & $:$ Módulo elástico del CFRP \\
$\varepsilon_{f u}:$ Deformación última del CFRP & & & & \\
\end{tabular}

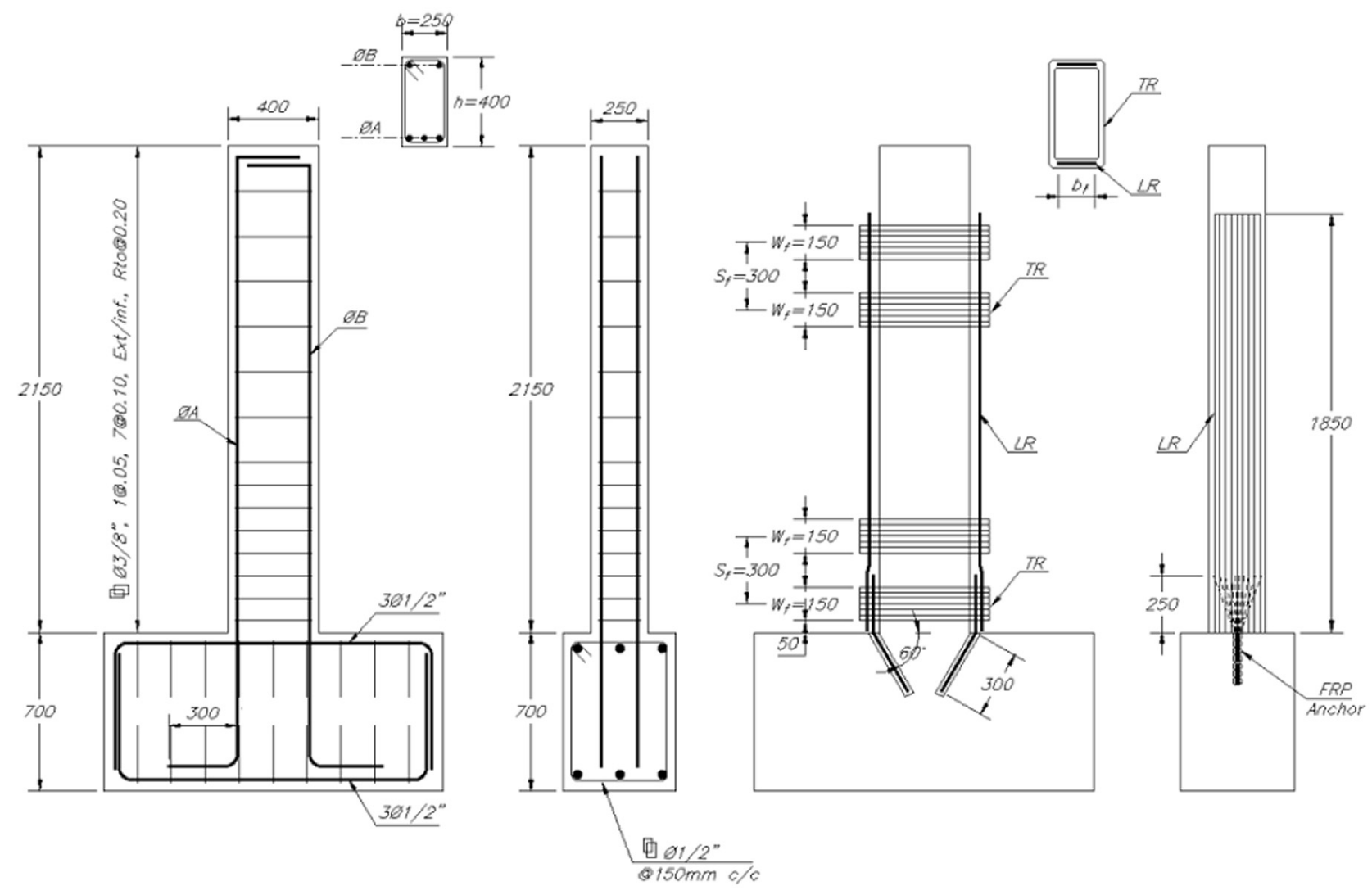

Figura 2. Detalles del refuerzo interno y externo de las vigas ensayadas Fuente: Elaboración propia 
Tabla 2. Características del refuerzo interno y externo

\begin{tabular}{|c|c|c|c|c|c|c|c|}
\hline ID & $\varnothing \mathrm{A}^{(+)}$ & $\varnothing \mathrm{B}^{(-)}$ & $n_{f}^{(+)}$ & $n_{f}(-)$ & $\begin{array}{c}\mathrm{LR} \\
b_{f}(\mathrm{~mm})\end{array}$ & $\begin{array}{l}\varnothing \text { FRP } \\
\text { anchor }\end{array}$ & $\begin{array}{c}\mathrm{TR} \\
W_{f} @ S_{f}\end{array}$ \\
\hline V-Control & $3 \varnothing 5 / 8 "$ & $3 \varnothing 5 / 8 "$ & - & - & - & - & $150 @ 300 \mathrm{c} / \mathrm{c}$ \\
\hline VE-01 & $3 \varnothing 5 / 8 "$ & $3 \varnothing 5 / 8 "$ & 1 & 1 & 200 & $\varnothing 3 / 4 ”$ & $150 @ 300 \mathrm{c} / \mathrm{c}$ \\
\hline VE-02 & $4 \varnothing 5 / 8 ”+1 \varnothing 1 / 2 "$ & $3 \varnothing 5 / 8 "$ & 3 & 3 & 200 & $\varnothing 1 "$ & $150 @ 300 \mathrm{c} / \mathrm{c}$ \\
\hline VE-03 & $4 \varnothing 5 / 8 "+1 \varnothing 1 / 2 "$ & $3 \varnothing 5 / 8 "$ & 1 & 1 & 200 & $\varnothing 3 / 4 "$ & $150 @ 300 \mathrm{c} / \mathrm{c}$ \\
\hline
\end{tabular}

\{-\} No hay valor

$n_{f} \quad$ : Número de capas de CFRP.

$b_{f} \quad$ : Ancho del refuerzo longitudinal CFRP (LR).

$W_{f}$ : Ancho del refuerzo transversal CFRP (TR).

$S_{f} \quad$ : Separación del refuerzo transversal de CFRP centro a centro (TR).

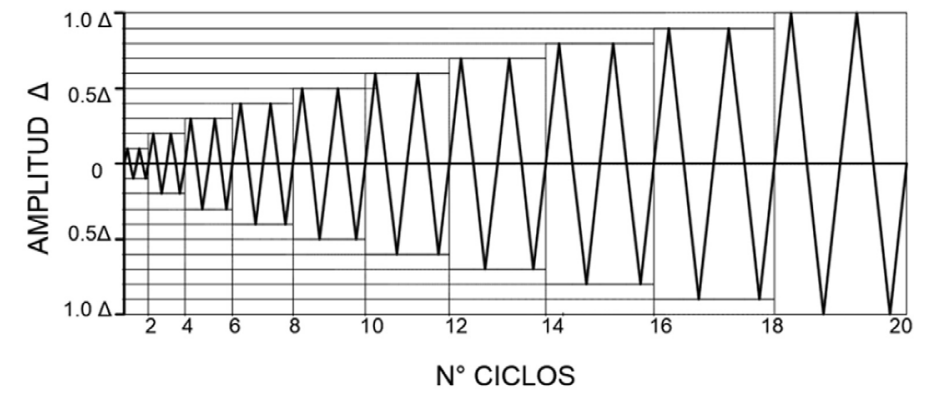

Figura 3. Protocolo de carga cíclica controlado por desplazamiento laterales Adaptado de FEMA 461 (2007)

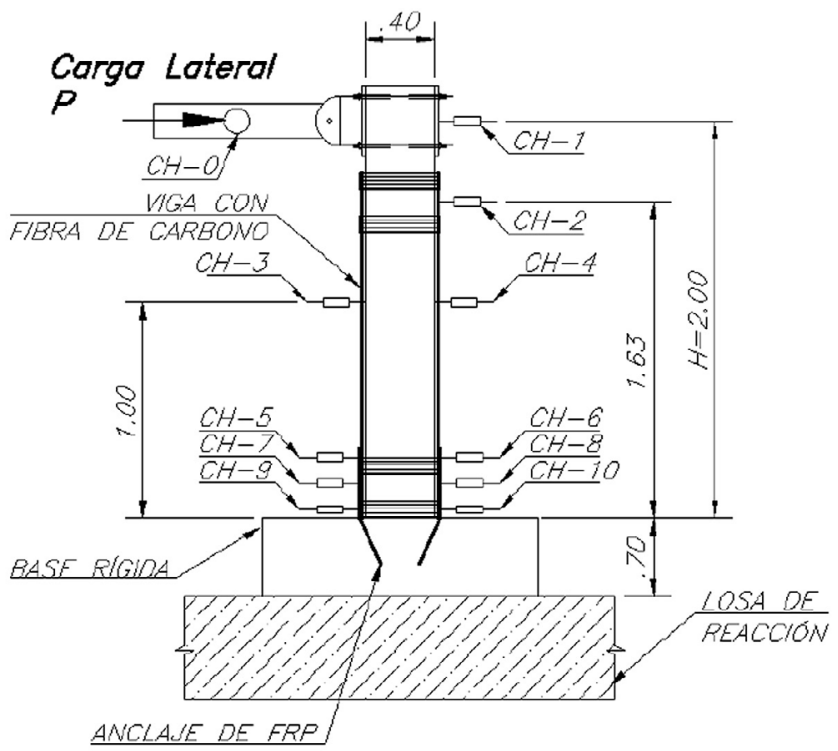

Figura 4. Instrumentación del ensayo de carga cíclica para el espécimen en voladizo (Unidades en 296 metros). Fuente: Elaboración propia 


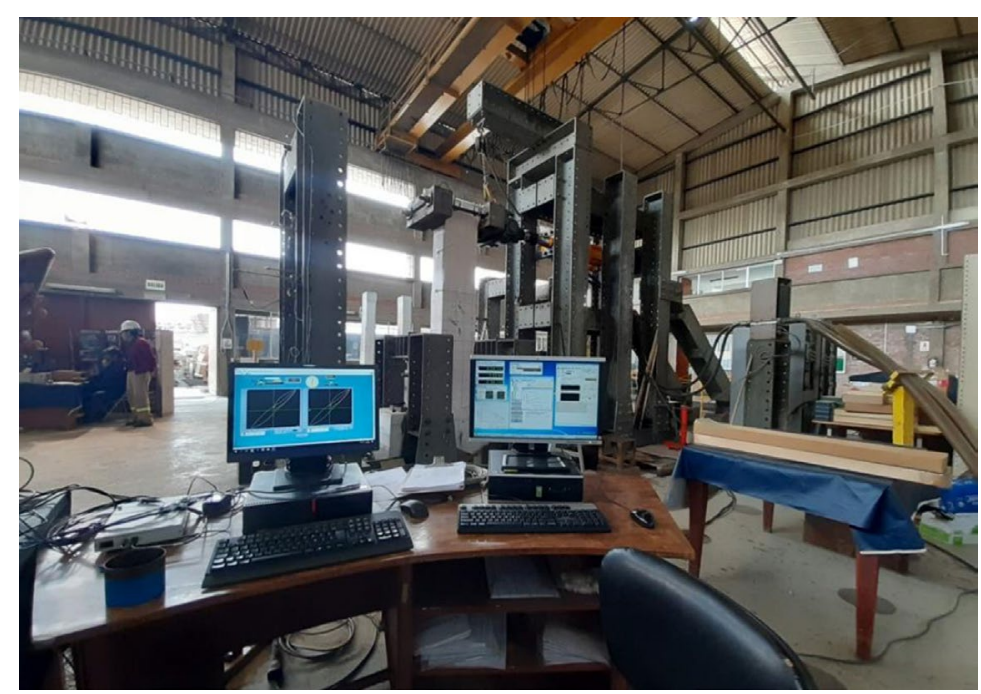

Figura 5. Vista general del ensayo en laboratorio de la PUCP Fuente: Elaboración propia
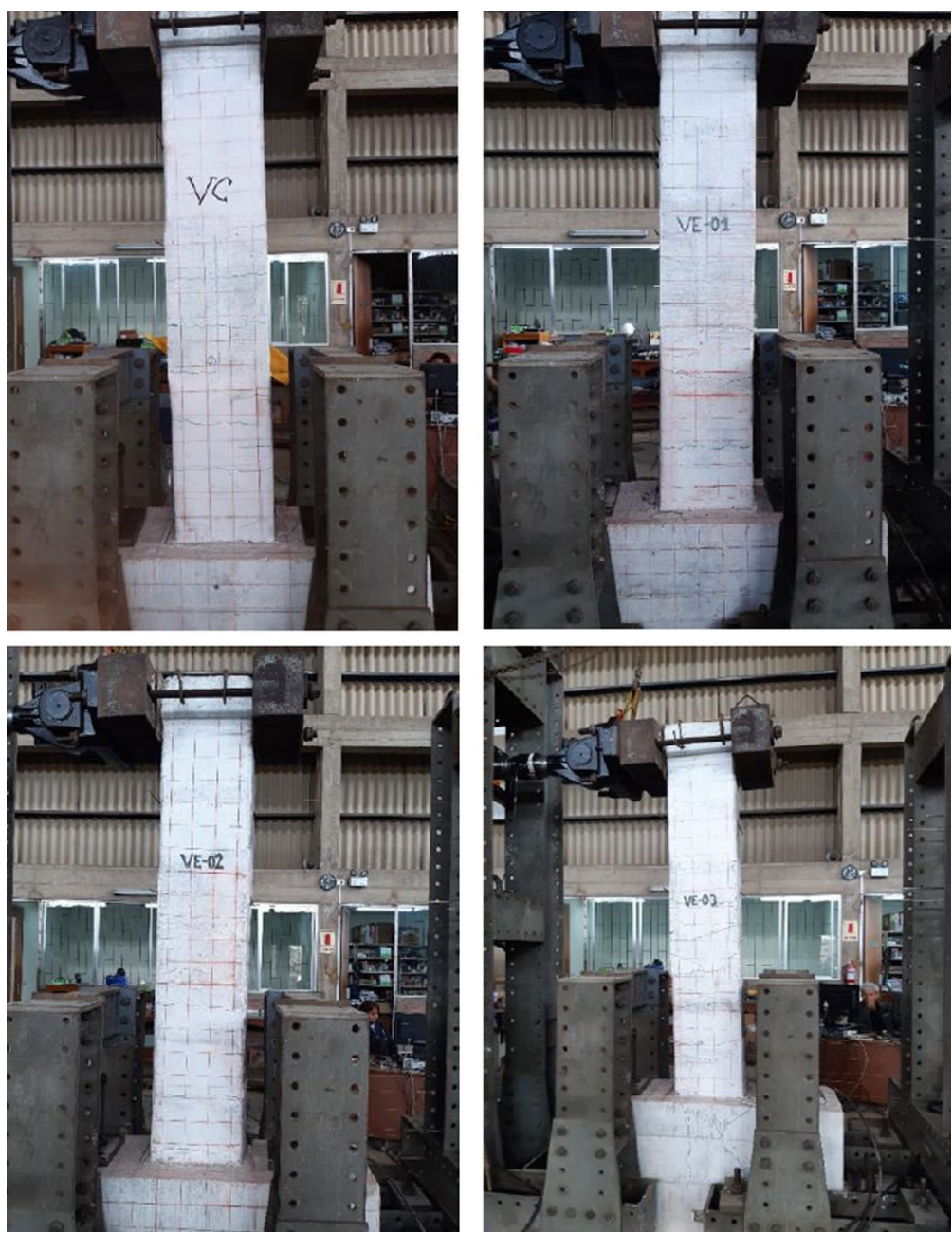

Figura 6. Vista de los especímenes durante el ensayo cíclico en el laboratorio de la PUCP. Fuente: Elaboración propia 


\section{RESISTENCIAS NOMINALES A FLEXIÓN}

Para calcular los valores de resistencias nominales a flexión de los especímenes, se utilizó el código ACI 318-14 (ACI, 2014) así como la guía de diseño ACI 440.2R-17 (ACI, 2017). La Tabla 3 muestra los valores calculados para la resistencia a flexión de la viga de CA y la resistencia a flexión de la viga de CA con refuerzo externo de bandas FRP de acuerdo con el código ACI 318 y la guía de diseño ACI 440.2R respectivamente. El superíndice (+) y (-) denota qué cara de la viga se tracciona y comprime respectivamente al inicio de la aplicación de las cargas cíclicas. La resistencia nominal de la viga de CA con refuerzo externo $\left(M_{n f}\right)$ se calculó de acuerdo con la Ec. 1 , considerando el aporte del acero de refuerzo $\left(M_{s}\right)$ y el aporte del FRP $\left(M_{f}\right)$ :

$$
M_{n f}=M_{s}+M_{f}
$$

Mientras que la Ec. 2 se utilizó para calcular la resistencia nominal reducida de la viga de CA con refuerzo externo $\left(\Phi \cdot M_{n f}\right)$, que incluye los factores de reducción $\Phi$ que varía de 0.65 a 0.90 según la deformación que experimente el acero de refuerzo, y $\psi=0.85$, el cual se aplica al FRP.

$$
\Phi \cdot M_{n f}=\Phi \cdot\left(M_{s}+\Psi \cdot M_{f}\right)
$$

El anclaje FRP fue diseñado mediante tres criterios de falla: por cono, por adherencia y por ruptura utilizando las ecuaciones propuestas por Kim y Smith (2010). Las principales variables utilizadas fueron: la profundidad de empotramiento del ancla $\left(h_{e f}\right)$, la resistencia a la compresión del concreto $\left(f^{\prime}{ }_{c}\right)$, el diámetro de la perforación $\left(d_{0}\right)$ y el esfuerzo de ruptura de las bandas $\operatorname{FRP}\left(f_{f u}\right)$.

$$
\begin{aligned}
& N_{m}=\min \cdot\left(N_{c c}, N_{c b}, N_{a r}\right) \\
& N_{c c}=9.68 \cdot h_{e f}^{1.5} \cdot \sqrt{{f^{\prime}}_{c}} \\
& N_{c b}=k \cdot \pi \cdot d_{0} \cdot h_{e f} \\
& N_{a r}=0.59 \cdot b_{f} \cdot t_{f} \cdot n_{f} \cdot f_{f u}
\end{aligned}
$$

Donde, $N_{c c}$ es el valor nominal de la falla del anclaje por cono de concreto (Ec. 4 ), $N_{c b}$ es el valor nominal de una falla por adherencia combinada con falla por cono (Ec. 5), $N_{a r}$ es la falla por ruptura del anclaje FRP (Ec. 6) y $k$ es un coeficiente que varía de 4.62 a 9.07, si $f_{c}$ ' es menor o mayor a $20 \mathrm{MPa}$ respectivamente (Kim \& Smith, 2010). Para los anclajes respectivos a los especímenes ensayados, se consideró que la capacidad del anclaje FRP, calculada mediante la Ec. 3, debe ser lo suficientemente resistente para soportar la exigencia que demande a tracción la banda FRP de refuerzo externo $\left(\Phi \cdot \psi \cdot N_{m}>T_{f}\right)$. Estos cálculos nominales también se muestran en la Tabla 3. 
Tabla 3. Resistencias nominales y reducidas de las vigas estudiadas

\begin{tabular}{|c|c|c|c|c|c|c|}
\hline ID & $\begin{array}{l}\Phi \cdot M_{n} \\
(\mathrm{kN}-\mathrm{m})\end{array}$ & $\begin{array}{c}M_{n f} \\
(\mathrm{kN}-\mathrm{m})\end{array}$ & $\Phi$ & $\begin{array}{l}\Phi \cdot M_{n f} \\
(\mathrm{kN}-\mathrm{m})\end{array}$ & $\begin{array}{c}T_{f} \\
(\mathrm{kN})\end{array}$ & $\begin{array}{c}\Phi \cdot \psi \cdot N_{m} \\
(\mathrm{kN})\end{array}$ \\
\hline V-Control ${ }^{(+)}$ & 69.36 & - & - & - & - & - \\
\hline VE-01 ${ }^{(+)}$ & 69.36 & 117.52 & 0.89 & 99.18 & 92.21 & 120.66 \\
\hline VE-02 ${ }^{(+)}$ & 102.02 & 176.19 & 0.70 & 116.25 & 159.90 & 169.71 \\
\hline VE-03 ${ }^{(+)}$ & 102.02 & 144.89 & 0.87 & 120.76 & 92.21 & 120.66 \\
\hline V-Control ${ }^{(-)}$ & 69.36 & - & - & - & - & - \\
\hline VE-01 $1^{(-)}$ & 69.36 & 117.52 & 0.89 & 99.18 & 92.21 & 120.66 \\
\hline VE-02 (-) & 69.36 & 117.52 & 0.71 & 94.27 & 159.90 & 169.71 \\
\hline VE-03 (-) & 69.36 & 117.52 & 0.89 & 99.18 & 92.21 & 120.66 \\
\hline$\{-\}$ & \multicolumn{6}{|l|}{ No hay valor } \\
\hline$\Phi$ & \multicolumn{6}{|c|}{ : Factor de reducción, controlado por deformación del acero de refuerzo. } \\
\hline $\begin{array}{l}\Phi \cdot M_{n} \\
M_{n f}\end{array}$ & \multicolumn{6}{|c|}{$\begin{array}{l}\text { : Resistencia a la flexión nominal reducida en viga sin refuerzo externo (ACI 318). } \\
\text { : Resistencia a la flexión nominal en viga con refuerzo externo. }\end{array}$} \\
\hline $\begin{array}{l}\Phi \cdot M_{n f} \\
T_{f} \\
\Phi \cdot \psi \cdot N\end{array}$ & \multicolumn{6}{|c|}{$\begin{array}{l}\text { : Resistencia a la flexión nominal reducida con refuerzo externo (ACI } 440.2 R) \text {. } \\
\text { : Capacidad nominal de la banda a la tracción a ser soportado por el anclaje. }\end{array}$} \\
\hline
\end{tabular}

\section{RESPUESTAS EXPERIMENTALES}

\subsection{Gráficos $P$ - $\Delta$}

Las Figuras 7 a 10 muestran las gráficas de carga versus desplazamiento lateral (P- $\Delta$ ) registrados durante el ensayo. Al comparar V-C y VE-01 (Figs. 7 y 8), se aprecia que la rigidez y la resistencia de la viga se incrementa de forma considerable al ser reforzada con una sola capa de FRP. Del mismo modo, al comparar VE-02 y VE-03 (Figuras 9 y 10) se aprecia que la fluencia ocurre para una carga similar actuante en ambas vigas, pero con un apreciable desplazamiento lateral mayor en la viga VE-02. Otra observación es que las vigas, cuyo refuerzo es simétrico en ambas caras, V-Control y VE-01, no exhiben una respuesta simétrica. La zona que inicialmente es comprimida ${ }^{(-)}$presenta mayores valores resistentes respecto a su contraparte ${ }^{(+)}$. 


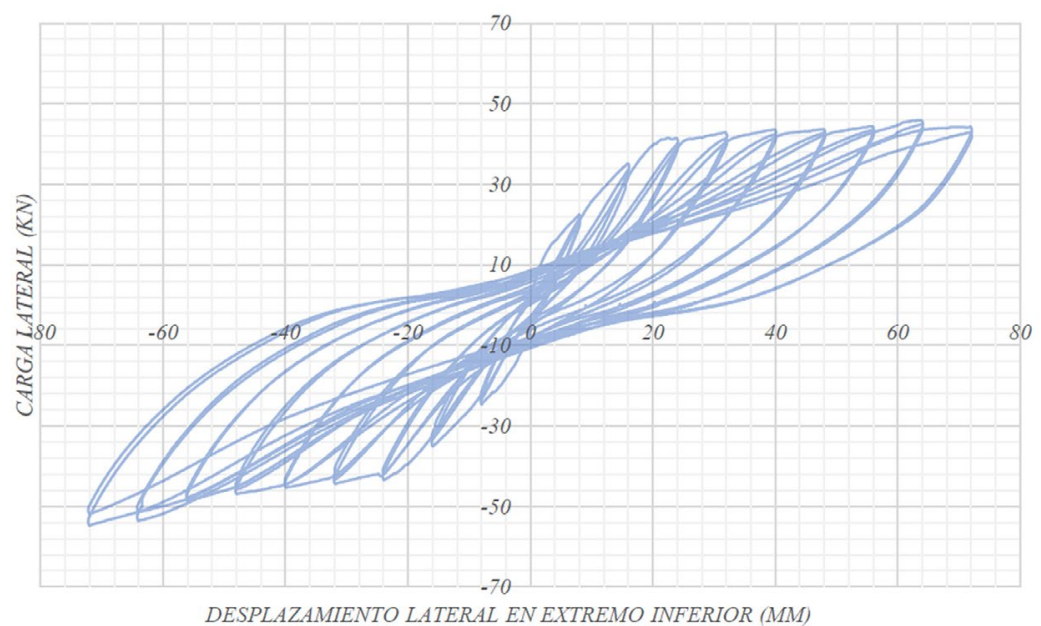

Figura 7. Respuesta de la viga VC sometida a cargas cíclicas Fuente: Elaboración propia

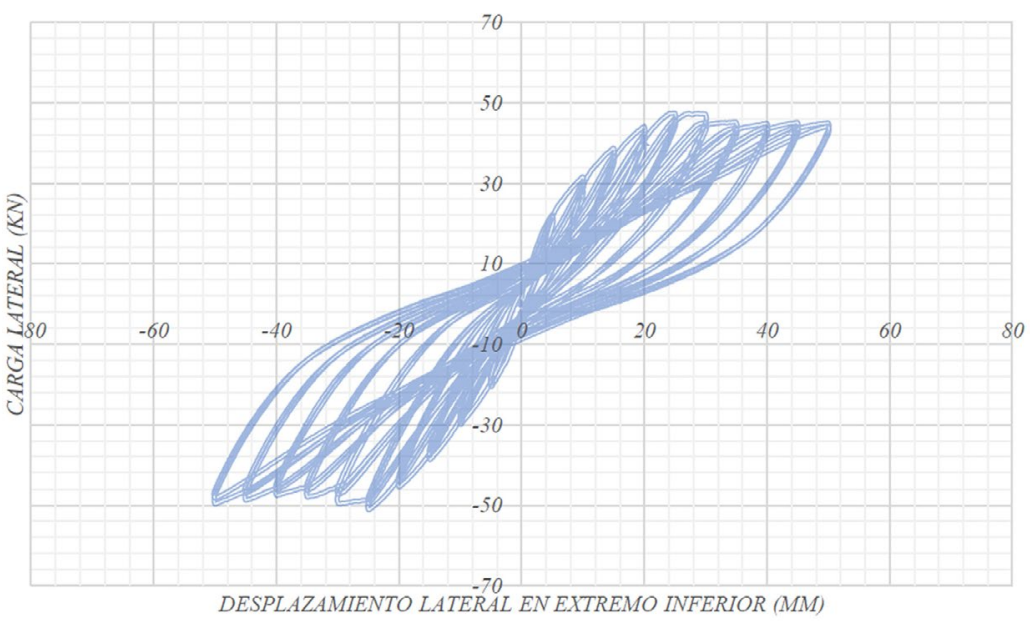

Figura 8. Respuesta de la viga VC sometida a cargas cíclicas Fuente: Elaboración propia

\subsection{Comportamiento experimental}

La viga de control tuvo un comportamiento histerético típico marcado por el deterioro gradual de la rigidez con el incremento de los ciclos de carga, mientras que los lazos de histéresis presentan una forma relativamente angosta, y finalmente la resistencia durante la etapa inelástica se mantiene sin degradación a pesar de las repeticiones de carga (Figura 7). Por otro lado, las vigas de CA con refuerzo externo CFRP, donde se resalta el hecho que la banda externa CFRP está empotrada en su extremo mediante un anclaje del mismo material, tienen un comportamiento similar al espécimen de concreto armado. Estas vigas reforzadas externamente (VE-01, VE-02 y VE-03) presentan una degradación más gradual de la rigidez respecto a la viga de control VC, además de exhibir lazos de histéresis más angostos que los del espécimen de control. Respecto a la resistencia, solo la 
viga VE-01 presenta una aparente degradación de la resistencia mientras que el resto de las vigas reforzadas presentan una resistencia más estable en la región inelástica (Figuras 8 a 10).

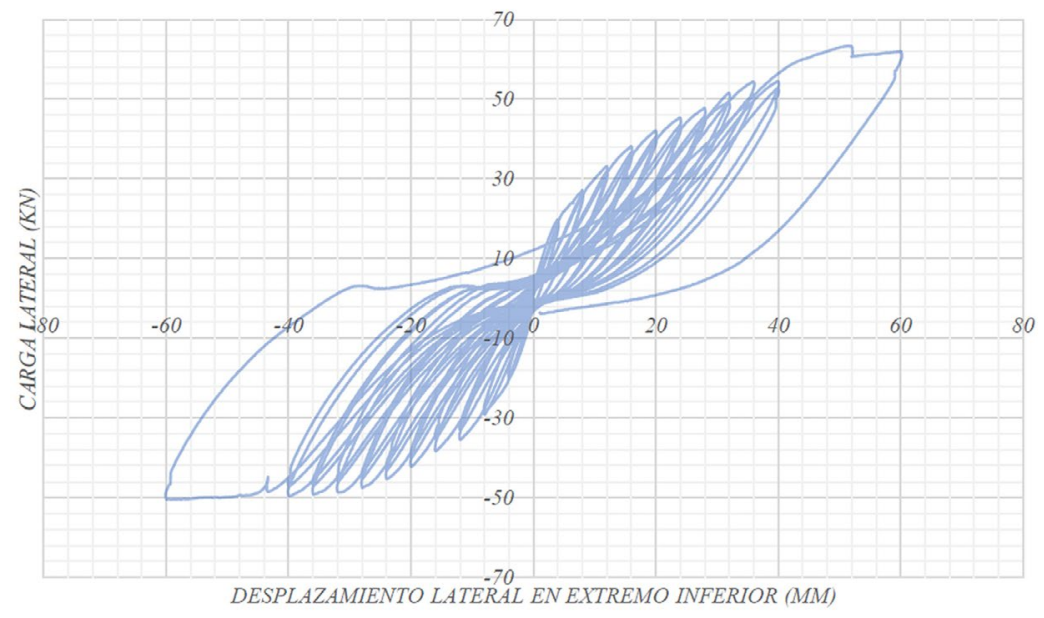

Figura 9. Respuesta de viga VE-02 sometida a cargas cíclicas Fuente: Elaboración propia

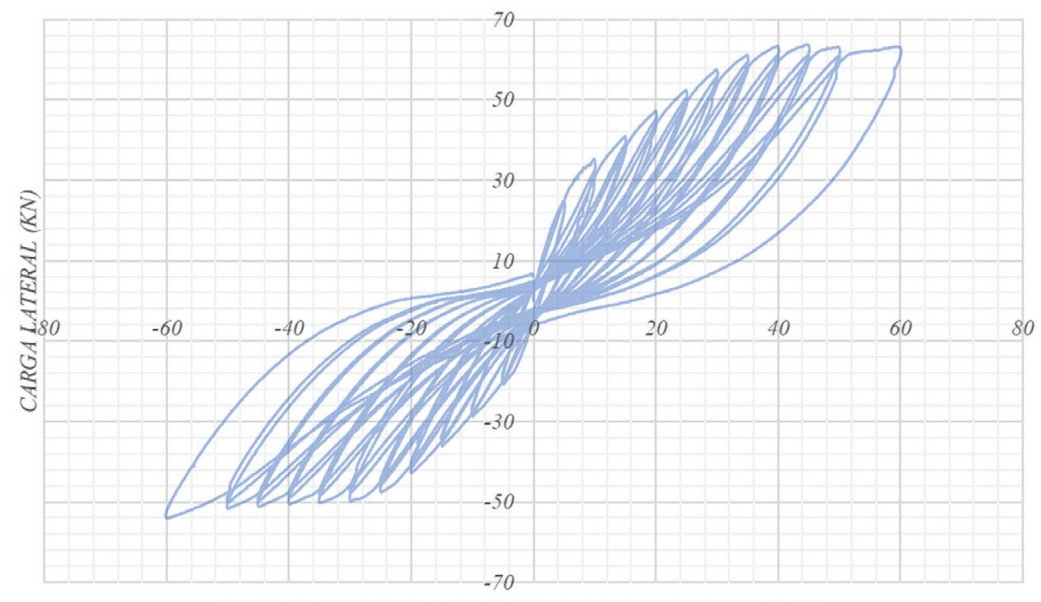

DESPLAZAMIENTO LATERAL EN EXTREMO INFERIOR (MM)

Figura 10. Respuesta de viga VE-03 sometida a cargas cíclicas

Fuente: Elaboración propia

\section{DISCUSION DE LOS RESULTADOS}

Se procedió a trazar la curva envolvente o primaria de cada uno de los gráficos $P$ - $\Delta$ discutidos en el apartado anterior. A partir de esta curva envolvente, se idealizó una curva trilineal que simula el comportamiento de la viga en cada una de las etapas de preagrietamiento, agrietamiento y fluencia de acero. Para ello, se empleó una técnica de compensación de áreas con el fin de ubicar aproximadamente los puntos correspondientes a la carga de agrietamiento $P_{c r}$, la carga de fluencia $P_{y}$ y la carga máxima del ensayo $P_{\text {máx }}$, así como también los valores de sus respectivos desplazamientos laterales $\Delta_{c r}, \Delta_{y}$ y $\Delta_{m a ́ x}$. Estas envolventes se muestran en las Figuras 11 a 14. 


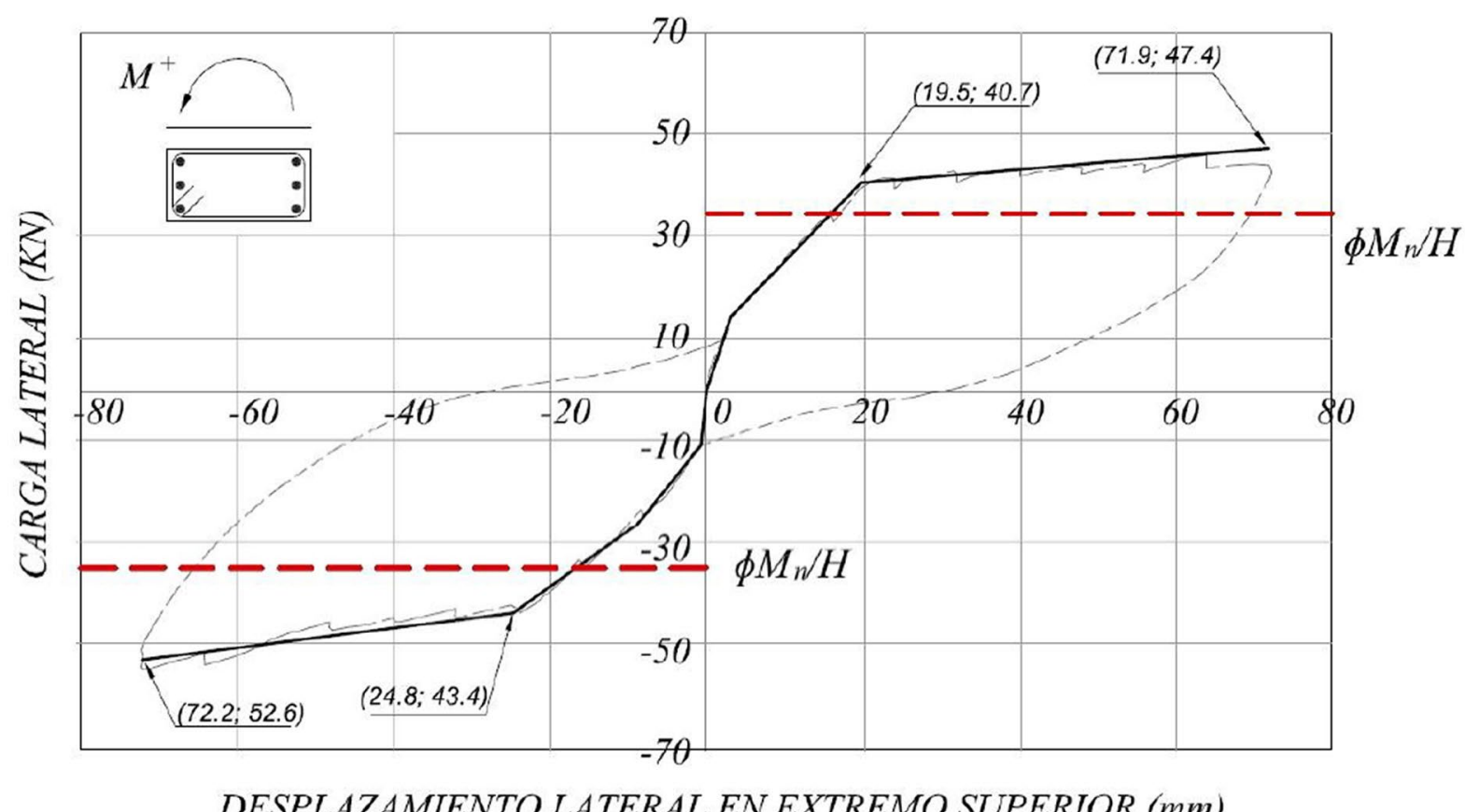

Figura 11. Curva envolvente y curva idealizada Tri-lineal de la viga VC Fuente: Elaboración propia

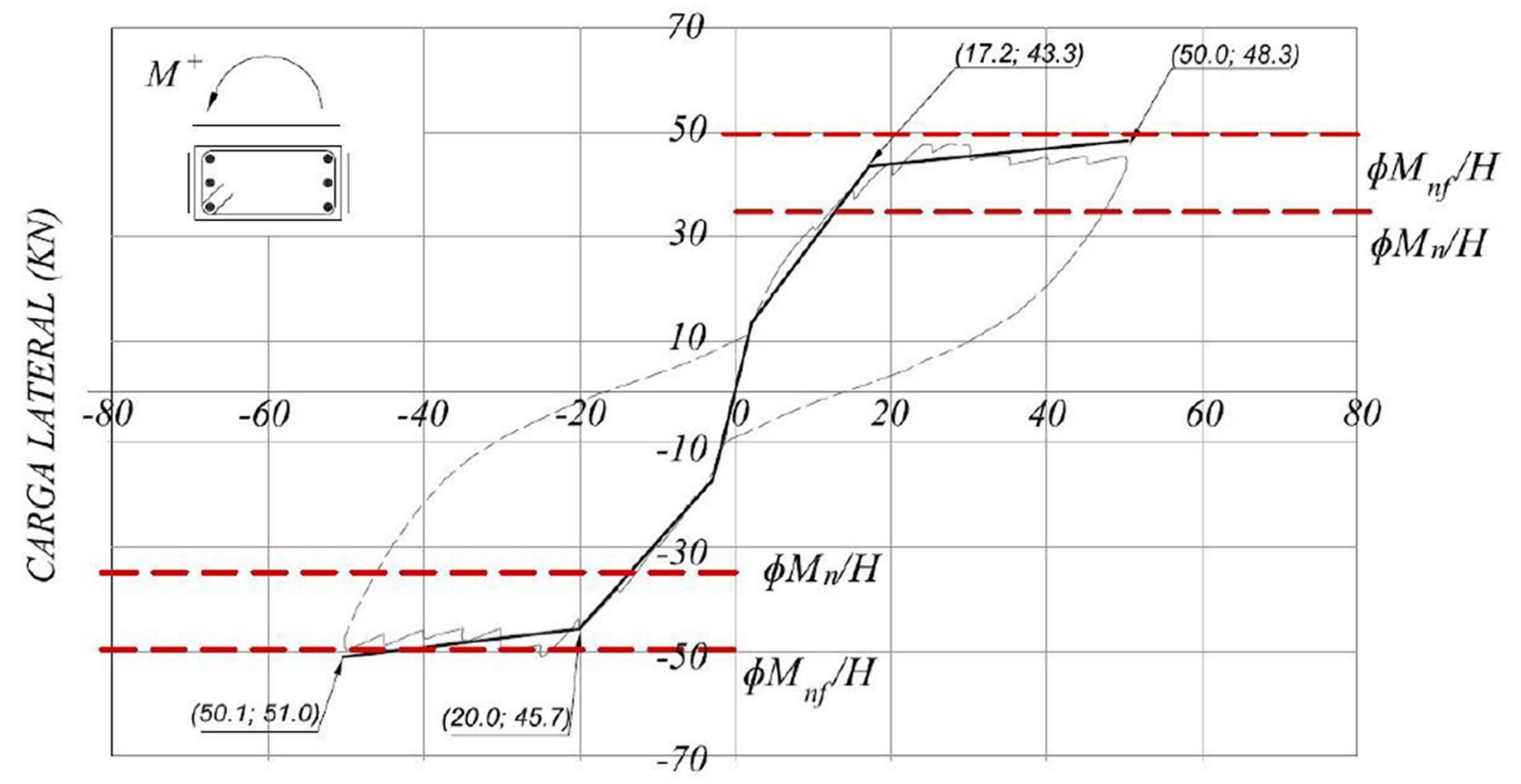

DESPLAZAMIENTO LATERAL EN EXTREMO SUPERIOR (mm)

Figura 12. Curva envolvente y curva idealizada Tri-lineal de la viga VE-01 Fuente: Elaboración propia 


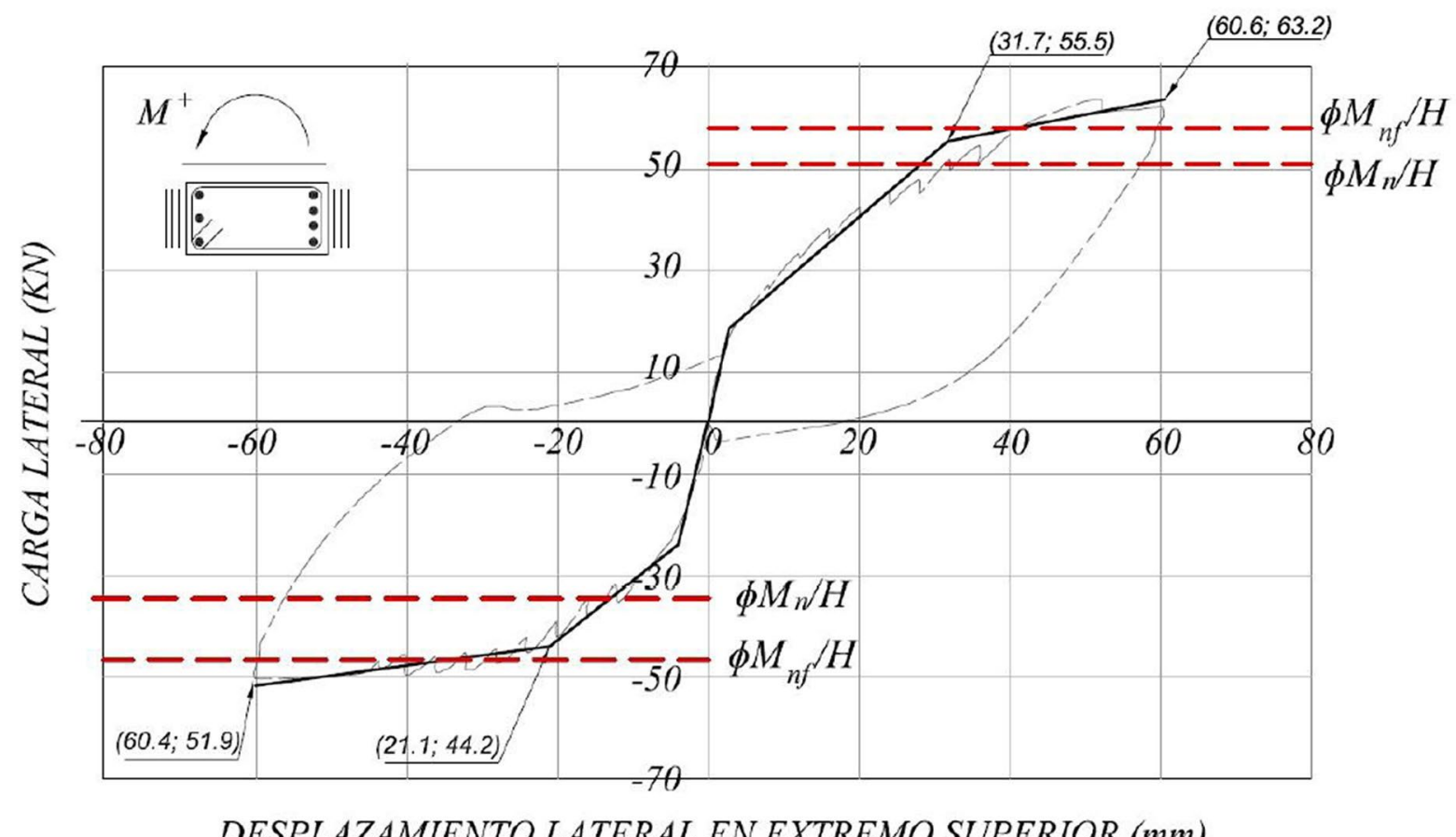

Figura 13. Curva envolvente y curva idealizada Tri-lineal de la viga VE-02 Fuente: Elaboración propia

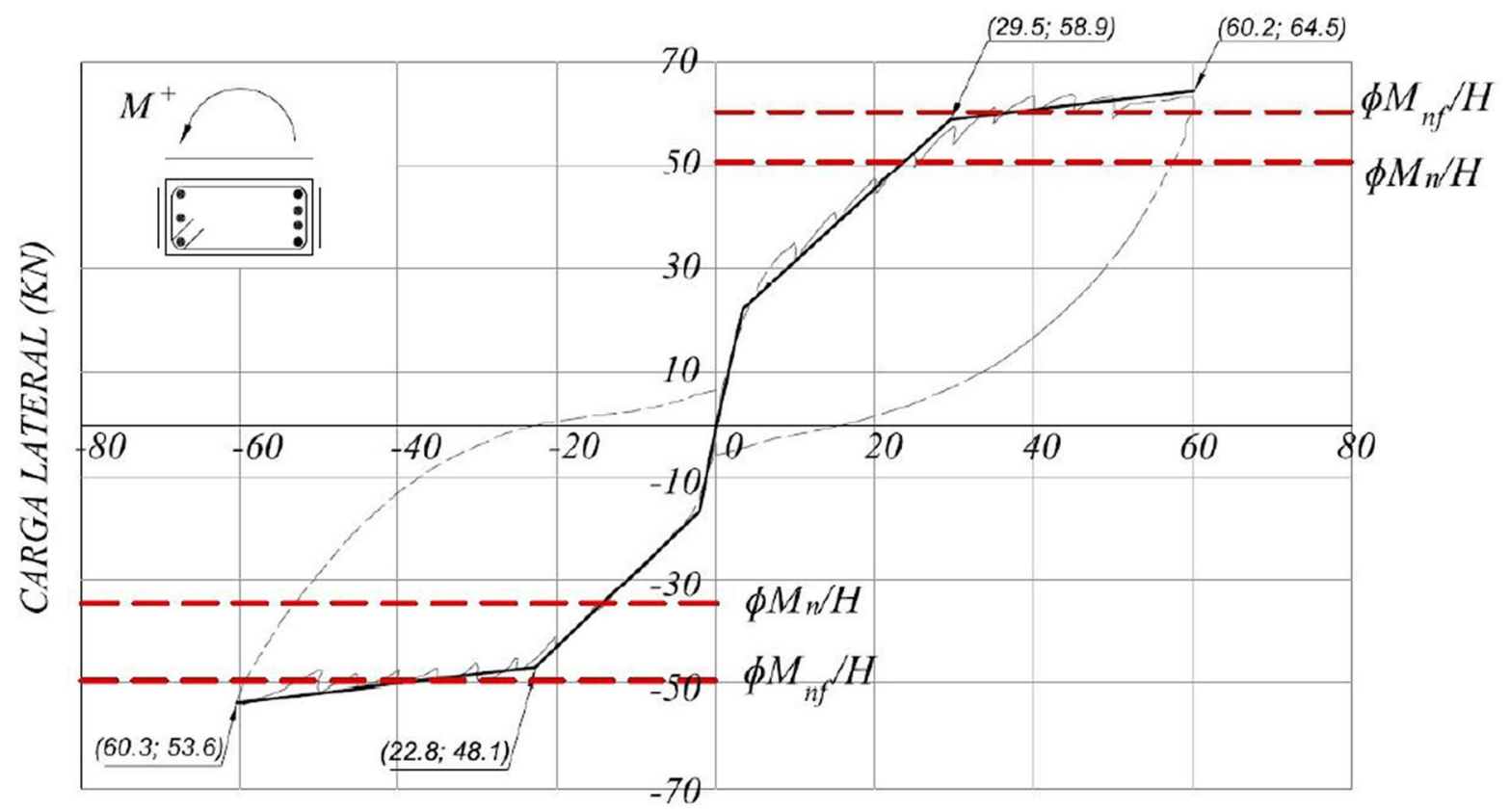

DESPLAZAMIENTO LATERAL EN EXTREMO SUPERIOR (mm)

Figura 14. Curva envolvente y curva idealizada Tri-lineal de la viga VE-03 Fuente: Elaboración propia 


\subsection{Resistencias flexurales}

La Tabla 4 presenta los valores registrados para la carga lateral durante la fluencia $\left(P_{y}\right)$ y la carga lateral máxima del ensayo $\left(P_{\max }\right)$. Cabe mencionar que, por precaución y cuidado de los equipos e instrumentos de laboratorio, los ensayos fueron detenidos antes de alcanzar la falla, con lo cual los valores de carga máxima $\left(P_{\text {máx }}\right)$ aquí indicados no representan necesariamente los valores últimos de falla esperados. La Tabla 4 presenta también la comparación del valor de fluencia experimental y el valor de resistencia nominal a flexión sin refuerzo externo y la comparación del valor máximo registrado en el ensayo y el valor de resistencia nominal a flexión de la viga con refuerzo externo.

Las Figuras 15 y 16 muestran que en aquellas vigas con el mismo reforzamiento interno y externo, VE-01 $1^{(+)}, \mathrm{VE}-01^{(-)}, \mathrm{VE}-02^{(-)}$y VE-03 $3^{(-)}$, la fluencia ocurre para valores de $M_{y}$ de $10 \%$ a 20 $\%$ mayores que los correspondientes valores de $M_{y}$ registrados en el espécimen de control. En la Figura 15 también se observa que los resultados de las vigas con diferente refuerzo externo CFRP e igual refuerzo interno (VE- $02^{(+)}$y VE- $03^{(+)}$), la fluencia ocurre en valores similares de $M_{y}$; $\sin$ embargo, los valores de desplazamiento lateral durante la fluencia $\Delta_{y}$ son moderadamente diferentes.

Tabla 4. Comparación de las resistencias nominales teóricas y experimentales

\begin{tabular}{ccccccc}
\hline ID & $\begin{array}{c}P_{y} \\
(\mathrm{kN})\end{array}$ & $\begin{array}{c}P_{\text {max }} \\
(\mathrm{kN})\end{array}$ & $\begin{array}{c}M_{y} \\
(\mathrm{kN}-\mathrm{m})\end{array}$ & $\begin{array}{c}M_{\text {máx }} \\
(\mathrm{kN}-\mathrm{m})\end{array}$ & $M_{y} / \Phi \cdot M_{n}$ & $M_{\max } / \Phi \cdot M_{n f}$ \\
\hline V-Control(+) & 40.7 & 47.4 & 81.4 & 94.8 & 1.17 & - \\
VE-01(+) & 43.3 & 48.3 & 86.6 & 96.6 & 1.25 & 0.97 \\
VE-02 (+) & 55.5 & 63.2 & 111.0 & 126.4 & 1.09 & 1.09 \\
VE-03 (+) & 58.9 & 64.5 & 117.8 & 129.0 & 1.15 & 1.07 \\
V-Control(-) & 43.4 & 52.6 & 86.8 & 105.2 & 1.25 & - \\
VE-01(-) & 45.7 & 51.0 & 91.4 & 102.0 & 1.32 & 1.03 \\
VE-02 (-) & 44.2 & 51.9 & 88.4 & 103.8 & 1.27 & 1.10 \\
VE-03 (-) & 48.1 & 53.6 & 96.2 & 107.2 & 1.39 & 1.08 \\
\hline
\end{tabular}

$\{-\} \quad$ No hay valor

$P_{y} \quad$ : Carga lateral en la fluencia registrada en el ensayo.

$P_{\text {máx }}^{y}$ : Carga lateral máxima registrada en el ensayo.

$M_{y}$ : Momento flector en la fluencia registrado en el ensayo.

$M_{\text {máx }}$ : Momento flector máximo registrado en el ensayo. 


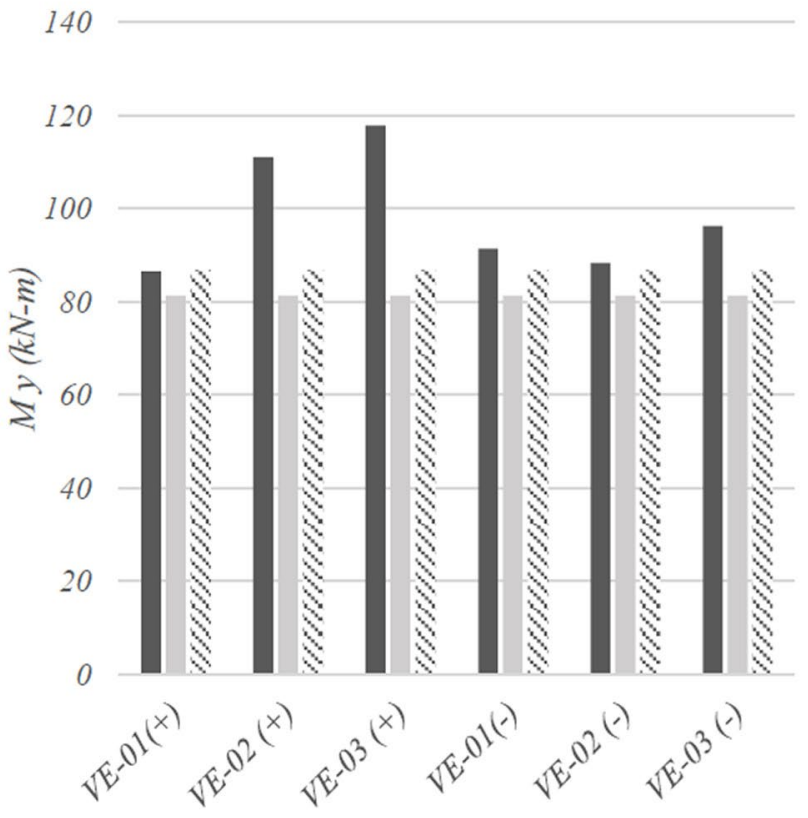

-Viga con FRP $\square$ V-Control (+) $\quad$ SV-Control (-)

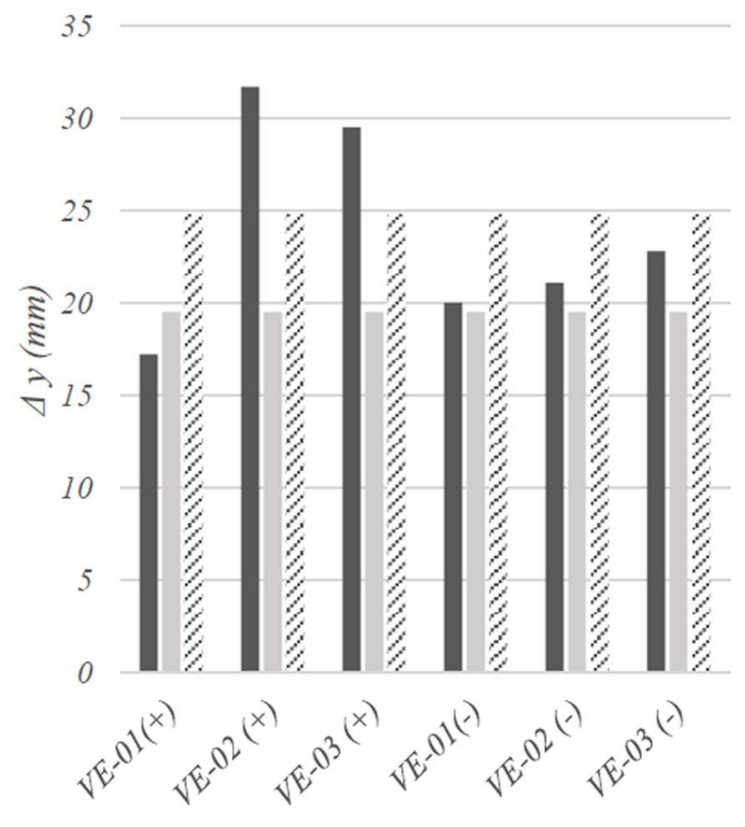

$\square$ Vigas con FRP $\square$-Control (+) $\%$ V-Control (-)

Figura 15. Comparación de resultados entre vigas reforzadas con CFRP y la viga de control Fuente: Elaboración propia

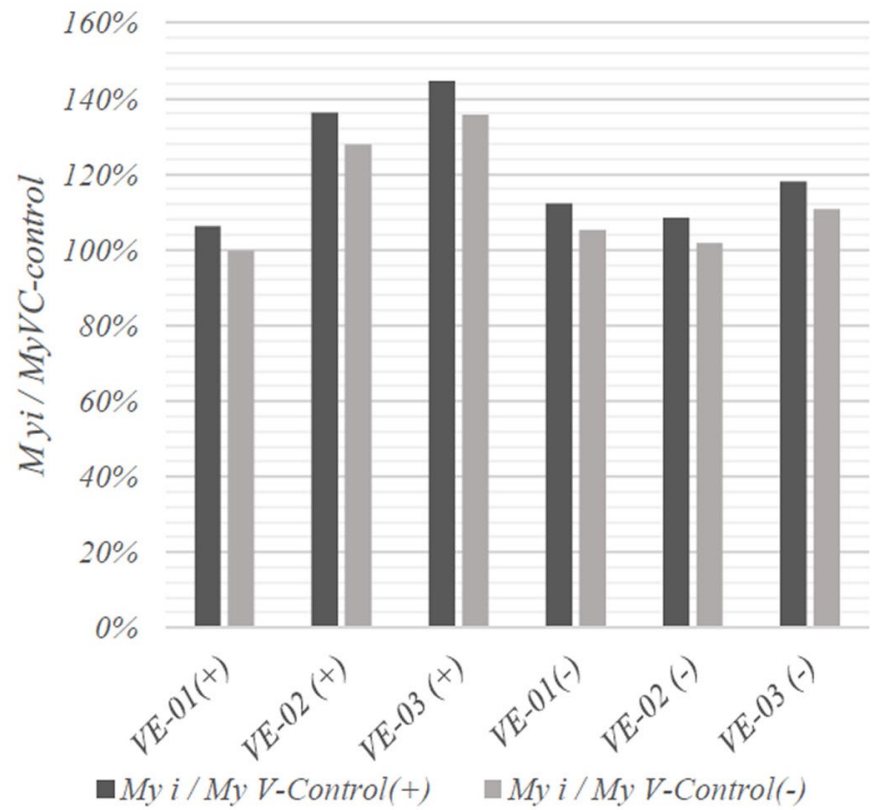

Figura 16. Comparación de los valores resistentes experimentales durante la fluencia entre las vigas reforzadas con CFRP y la viga de control. Fuente: Elaboración propia 
Tabla 5. Desplazamientos laterales experimentales y ductilidad de desplazamiento lateral parcial

\begin{tabular}{lccc}
\hline \multicolumn{1}{c}{ ID } & $\Delta_{y}(\mathrm{~mm})$ & $\Delta_{\text {máx }}(\mathrm{mm})$ & $\mu^{\prime}{ }^{\prime}$ \\
\hline V-Control $^{(+)}$ & 19.5 & 71.9 & 3.69 \\
VE-01( $^{(+)}$ & 17.2 & 50.0 & 2.91 \\
VE-02 $^{(+)}$ & 31.7 & 60.6 & 1.91 \\
VE-03 $^{(+)}$ & 29.5 & 60.2 & 2.04 \\
V-Control $^{(-)}$ & 24.8 & 72.2 & 2.91 \\
VE-01( $^{(-)}$ & 20.0 & 50.1 & 2.51 \\
VE-02 $^{(-)}$ & 21.1 & 60.4 & 2.86 \\
VE-03 $^{(-)}$ & 22.8 & 60.3 & 2.64 \\
\hline
\end{tabular}

$\Delta_{y} \quad$ : Deflexión lateral durante la fluencia

$\Delta_{\text {máx }}$ : Deflexión lateral máxima registrada en el ensayo.

$\mu_{\Delta}^{\prime} \quad$ : Ductilidad de desplazamiento lateral parcial.

\subsection{Desplazamientos laterales}

La Tabla 5 muestra los valores de desplazamiento lateral $(\Delta)$, registrado a una altura $H$ de la base. También se muestra el cálculo de la ductilidad de desplazamiento lateral parcial, el cual se obtiene como el cociente de la deflexión lateral máxima y la deflexión lateral durante la fluencia. Se aprecia que, pese a la simetría de refuerzo de acero y FRP, las vigas V-Control y VE-01 exhiben diferentes ductilidades, siendo el lado donde inicia la tracción ${ }^{(+)}$el que exhibe mayor valor de ductilidad parcial. Al evaluar los valores de desplazamiento lateral durante la fluencia $\Delta_{y}$, no se observa una clara tendencia en los valores registrados para los especímenes reforzados y el espécimen de control. Sin embargo, para secciones de viga de CA con el mismo refuerzo interno y externo $\left(\mathrm{VE}-01^{(+)}, \mathrm{VE}-01^{(-)}\right.$y $\left.\mathrm{VE}-03^{(-)}\right)$, los valores de desplazamiento lateral (cuando la fluencia es alcanzada) son mayores en el lado donde se inicia la compresión, es decir $\Delta y^{(-)}>\Delta y^{(+)}$para vigas con el mismo reforzamiento, cumpliéndose la siguiente relación de ductilidad parcial: $\mu^{\prime} \Delta^{(-)}$ $<\mu^{\prime} \Delta^{(+)}$.

\section{CONCLUSIONES Y RECOMENDACIONES}

El presente estudio centró la discusión de los resultados obtenidos del ensayo experimental de cuatro especímenes de CA: uno de ellos sin refuerzo externo y los otros tres con refuerzo externo CFRP. El refuerzo fue calculado utilizando la guía de diseño ACI 440.2R y cuyos anclajes de CFRP 
se diseñaron a partir de los valores recomendados en el estudio estadístico de Kim y Smith (2010). De este modo se desprenden las siguientes conclusiones:

a) Los especímenes de CA reforzados y ensayados lograron desarrollar la capacidad nominal a flexión según la guía ACI 440.2R (ACI, 2017).

b) Las bandas externas de CFRP colocadas en los especímenes de CA reforzados desarrollaron apropiadamente su aporte a la resistencia flexural teórica, incluso bajo condiciones cíclicas y con uno de sus extremos anclados en el concreto mediante anclajes CFRP.

c) Los especímenes de CA, externamente reforzadas con CFRP empotrada a la base con anclajes CFRP, exhibieron lazos de histéresis similares al del espécimen de control bajo condiciones de ensayo cíclico cuasi-estático (Item 4.2).

d) No se produjo la falla del sistema ni en la banda CFRP ni en el anclaje CFRP para la amplitud objetivo seleccionada, obteniéndose los valores de capacidad nominal a flexión del espécimen.

e) El valor experimental de la resistencia a la fluencia de las vigas de CA reforzadas con CFRP se incrementó entre $10-20 \%$ respecto a la viga de CA de control para las vigas con el mismo acero de refuerzo.

f) El valor de la carga resistente durante la fluencia fue similar para las vigas con igual cuantía de refuerzo interno, independiente del número de capas de refuerzo CFRP externo. Además, no se observó una tendencia en el valor de desplazamiento lateral durante la fluencia entre los especímenes reforzados con CFRP y los especímenes de control.

Finalmente, se recomienda considerar mayores valores de desplazamiento lateral total para ensayos de carga cíclica en especímenes similares con el objeto de inducir fallas en el sistema resistente a flexión y obtener los valores últimos de resistencia y desplazamiento lateral que permitan evaluar la resistencia última y la ductilidad global. Finalmente, se recomienda realizar un mayor número de ensayos experimentales con la finalidad de verificar el comportamiento favorable de las bandas de CFRP empotradas con anclajes del mismo material y sometidas a solicitaciones cíclicas (carga y descarga). Tales estudios se podrían enfocar en parámetros como los modos de falla, la longitud y el diámetro del empotramiento del anclaje CFRP, entre otros.

\section{AGRADECIMIENTOS}

Los autores desean expresar su profundo agradecimiento a la Dirección del Programa de Maestría en Ingeniería Civil de la Escuela de Posgrado y a la Sección de Ingeniería Civil de la Pontificia Universidad Católica del Perú (PUCP). Así como también a la empresa Top Consult Ingeniería SAC por su patrocinio en la donación de las fibras de carbono que fueron utilizadas en la investigación. 


\section{REFERENCIAS}

American Concrete Institute (ACI Committee 318). (2014). Building code requirements for structural concrete (ACI-318-14) and commentary (ACI 318R-14). Farmington Hills, Michigan: American Concrete Institute.

American Concrete Institute (ACI Committee 440). (2017). Guide for the Design and Construction of Externally Bonded FRP Systems for Strengthening Concrete Structures (ACI 440-2R-17). Farmington Hills, MI: American Concrete Institute.

Bazan, J.L. \& Fernández-Dávila G., V.I. (2019). Evaluación de la sobrerresistencia a la flexión de vigas de concreto armado reforzadas con bandas FRP. XII Congreso Chileno de Sismología e Ingeniería Sísmica. Chile.

Binici B., Ozcebe G. \& Ozcelik R. (2007). Analysis and design of FRP composites for seismic retrofit of infill walls in reinforced concrete frames. Composites Part B Eng, 38, 575-583.

Breña, S., Bramblett, M., Benouaich, M., Wood, S. \& Kreger, M. (2001). Use of carbon fiber reinforced polymer composites to increase the flexural capacity of reinforced concrete beams (Research Report no. 1776-1). Austin: The University of Texas.

Federal Emergency Management Agency, FEMA 461 (2007). Interim Testing Protocols for Determining the Seismic Performance Characteristics of Structural and Nonstructural Components. Federal Emergency Management Agency, USA.

Kim, SJ. \& Smith, ST. (2009). Behaviour of handmade FRP anchors under tensile load in uncracked concrete. Advances in Structural Engineering, 12(6), 845-865.

Kim, SJ. \& Smith, ST. (2010). Pullout Strength Models for FRP Anchors in Uncracked Concrete. Journal of composites for construction, 14, 406-414.

Landesmann, A., Seruti, C.A. \& Batista, E.d.M. (2015). Mechanical Properties of Glass Fiber Reinforced Polymers Members for Structural Applications. Materials Research, 18(6), 1372-1383.

Mostofinejad, D. \& Khozaei, K. (2015). Effect of GM patterns on ductility and debonding control of FRP sheets in RC strengthened beams. Construction and Building Materials, 93, 110-120.

Noorunnisa Khanam, P., Abdul Khalil, HPS., Jawaid, M., Ramachandra Reddy, G., Surya Narayana, C. \& Venkata Naidu, S. (2010). Sisal/carbon fiber reinforced hybrid composites: tensile, flexural and chemical resistance properties. Journal of Polymers and the Environment, 18(4), 727-733.

Orton S., Jirsa J. \& Bayrak O. (2008). Design Considerations of Carbon Fiber Anchors. Journal of Composites for Construction ASCE, 12(6), 608-616.

Samaniego, J.D. (2020). Análisis experimental del comportamiento de vigas de concreto armado reforzadas con bandas de CFRP bajo acciones cíclicas en reversa "Quasi-estática" (Tesis de Maestría en Ingeniería Civil en progreso). Pontificia Universidad Católica del Perú.

Tan, K. H. \& Mathivoli, M. (1998). Behavior of preloaded reinforced concrete beams strengthened with carbon fiber sheets. Fourth International Symposium on fiber reinforced Polymer for Reinforced Concrete Structures. Baltimore.

White, T. W., Soudki, K. A. \& Erki, M. A. (1999). Analytical Modeling of Reinforced Concrete Beams Strengthened with Carbon Fiber Reinforced Polymer Laminates Subjected to High Strain Rates. Supplement-ACI-SP-188 on Non-Metallic (FRP) Reinforcement for Concrete Structures. Québec: American Concrete Institute. 